\title{
Comparações florísticas e estruturais entre comunidades de palmeiras em fragmentos de floresta primária e secundária da Área de Proteção Ambiental Raimundo Irineu Serra - Rio Branco, Acre, Brasil
}

\begin{abstract}
Anelena Lima de CARVALHOํㅗㄹ Evandro José Linhares FERREIRA², Joanna Marie Tucker LIMA³
RESUMO

O presente estudo compara a composição e estrutura das comunidades de palmeiras da Área de Proteção Ambiental Raimundo Irineu Serra - APARIS, localizada no perímetro urbano do Município de Rio Branco-Acre. Foram selecionadas três áreas de floresta secundária em estágios sucessionais distintos: 7,5 anos, 27,5 anos, 37,5 anos de idade, e um fragmento de floresta primária. Em cada área foram instaladas cinco parcelas de $20 \mathrm{X} 20 \mathrm{~m}$, onde foram analisadas a composição florística, estrutura horizontal e estrutura populacional das palmeiras. Foram identificados 1.034 indivíduos, incluídos em 12 gêneros e 19 espécies de palmeiras. A área de floresta primária apresentou maior diversidade. $\mathrm{Na}$ análise da estrutura populacional de cada área, comprovamos a existência de uma escassez de plântulas ( $\leq 50 \mathrm{~cm}$ de altura) e adultos reprodutivos. A fragmentação alterou a composição e diminuiu a riqueza e a diversidade de palmeiras na área da APARIS, enquanto, está favorecendo a dominância de certas espécies como $A$. phalerata.
\end{abstract}

PALAVRAS-CHAVE: Arecaceae, composição florística, estrutura populacional, fragmentos florestais, floresta secundária

\section{Floristic and structural comparisons among palm communities in primary and secondary forest fragments of the Raimundo Irineu Serra Environmental Protection Area - Rio Branco, Acre, Brazil}

\section{ABSTRACT}

This study compares the composition and structure of palm communities in fragments of secondary and primary forest within the Raimundo Irineu Serra Environmental Protection Area (APARIS), located at the urban perimeter of Rio Branco, Acre. To evaluate the palm communities, we selected secondary forest areas belonging to three distinct successional stages: 7.5 years; 27.5 years, 37.5 years, and a primary forest fragment. In each forest type we installed five 20 x $20 \mathrm{~m}$ plots, where we analyzed floristic composition, vegetation structure, and population demography of all palm species (Arecaceae). In all, we identified 1034 palm individuals, including 12 genera, 19 species. Primary forest exhibited the greatest palm diversity. Structural analysis of each area revealed a scarcity of seedlings $(\leq 50 \mathrm{~cm}$ tall $)$ and reproductive adults. Fragmentation altered the composition and decreased the richness and diversity of palms within the APARIS, while at the same time, favoring the dominance of certain species, such as $A$. phalerata.

KEY WORDS: Arecaceae, floristic composition, population structure, forest fragments, secondary forest

\footnotetext{
1 Instituto Nacional de Pesquisas da Amazônia.E-mail:anelenalcarvalho@yahoo.com.br

2 Instituto Nacional de Pesquisas da Amazônia.E-mail:evandroferreira@yahoo.com

${ }^{3}$ Universidade da Flórida.E-mail: jmtucker@ufl.edu
} 


\section{INTRODUÇÃO}

O processo de desenvolvimento ao qual a Amazônia vem sendo submetida tem provocado a formaçáo de mosaicos de fragmentos florestais cujas áreas podem ser de diversas ordens de magnitude (Bierregaard et al., 1992). A maioria destes fragmentos é pequeno e muito alterado, e geralmente ficam localizados em áreas não aptas para atividades econômicas lucrativas, devido à topografia acentuada, solos pobres, ou de difícil acesso.

Conforme as paisagens florestais tornam-se fragmentadas, as populaçóes das espécies são reduzidas, os padrôes de migração e dispersáo sáo alterados e os habitats tornam-se expostos à condiçóes externas adversas anteriormente inexistentes, o que resulta, em última análise, numa deterioração da diversidade biológica ao longo do tempo (Tilman et al., 1994; Terborgh et al., 1997).

As mudanças ecológicas que ocorrem em função do isolamento do fragmento proporcionais à área do fragmento são definidas como efeito de área. Além disso, ocorrem também os efeitos de borda causados por gradientes diferenciados de mudanças fisícas e bióticas próximos às bordas florestais proporcionais à distância da borda mais próxima (Lovejoy et al., 1986; Murcia, 1995). Ambos operam conjuntamente, pois quanto menor o tamanho de um fragmento florestal maior é a razão borda/área, tornando fragmentos menores mais sujeitos a maiores intensidades dos efeitos de borda (Zuidema et al., 1996).

As espécies que ocorrem naturalmente em baixa densidade podem sofrer considerável redução do tamanho populacional em fragmentos pequenos tornando-se mais vulneráveis à extinçâoo local devido a eventos estocásticos demográficos, catastróficos e genéticos (Shafer, 1981).

Entre as famílias botânicas susceptíveis a essas alteraçóes podemos citar a família Arecaceae, uma das mais importantes da regiáo amazônica, em razão de sua ampla distribuição, abundância nos mais diversos ecossistemas e, principalmente, sua diversidade de usos (Ferreira, 2005). A diminuição de sua densidade e perda de espécies, nos fragmentos florestais, pode desencadear mudanças imprevisíveis na estrutura da floresta e na cadeia alimentar da fauna nativa local, diminuindo, conseqüentemente, o valor biológico dessas reservas (Scariot, 1998).

O presente estudo teve como objetivo conhecer e comparar a composição e a estrutura de comunidades de palmeiras em fragmentos de floresta primária e secundária, localizados no perímetro urbano da cidade de Rio Branco AC. Esperamos que esses resultados possam contribuir para o melhor entendimento do processo de estabelecimento das comunidades de palmeiras, depois do desmatamento e uso da terra, permitindo que se faça uma avaliação do comportamento destas comunidades no contexto atual de desmatamento na Amazônia.

\section{MATERIAL E MÉTODOS}

\section{Área de estudo e delineamento amostral}

O estudo foi realizado na Área de Proteção Ambiental Raimundo Irineu Serra - APARIS, localizada na Vila Irineu Serra, no perímetro urbano do Município de Rio Branco. A APARIS possui uma área de 909 hectares, das quais $30 \%$, ou aproximadamente 270 hectares, são cobertas por fragmentos florestais em diversos estágios de regeneração (Figura 1).

$\mathrm{O}$ início da colonização da região onde a APARIS está localizada ocorreu há mais de 50 anos, quando o Governo do Estado doou a mesma ao Sr. Raimundo Irineu Serra, fundador e líder da doutrina religiosa conhecida como Santo Daime, que estabeleceu no local uma comunidade de seguidores. Ao longo dos anos, a área foi fracionada em pequenos lotes para abrigar novos membros da doutrina. Os lotes foram utilizados para a prática da agricultura de subsistência, criação de pequenos animais e pecuária em pequena escala. Como resultado, a cobertura florestal original do local foi gradualmente alterada, resultando na formação de diversos fragmentos florestais (PMRB, 2005).

O avanço da urbanização e da especulação imobiliária no entorno da área ocupada pelos seguidores da doutrina daimista a partir dos anos 90 aumentou a pressão sobre os remanescentes florestais, incluindo a mata ciliar do igarapé São Francisco, que corta a área da APARIS e se constitui no mais importante meio de drenagem da área urbana de Rio Branco. Estes fatos levaram a Prefeitura de Rio Branco a decretar, em 2005, a criação de uma Área de Proteção Ambiental (APA) englobando toda a regiáo ocupada pela comunidade daimista e a parte do igarapé São Francisco que corta a área.

A presença de moradores no interior da APARIS, prevista no Sistema Nacional de Unidades de Conservação - SNUC (Lei No 9.985, de 18 de julho de 2000), sugere que o controle sobre o processo de ocupação da área e a sustentabilidade do uso de seus recursos naturais são os maiores desafios que os gestores da unidade enfrentarão para garantir a conservação da mesma no longo prazo.

A temperatura média anual da regiáo varia entre 24,7 e $25,1^{\circ} \mathrm{C}$ com a precipitaçáo média anual entre 1.877 e 1.982 $\mathrm{mm}$, e sua vegetaçáo é classificada como Floresta Ombrófila Aberta com Bambu e Palmeiras (Acre, 2000).

Foram selecionadas áreas de floresta secundária (aqui denominadas de capoeira), em três estágios sucessionais com idade média de 7,5 anos, 27,5 anos, 37,5 anos, e uma área de floresta primária (Figura 1). Para estimar a idade das áreas, foram considerados o tempo de abandono das mesmas informados pelos moradores e o estádio sucessional 


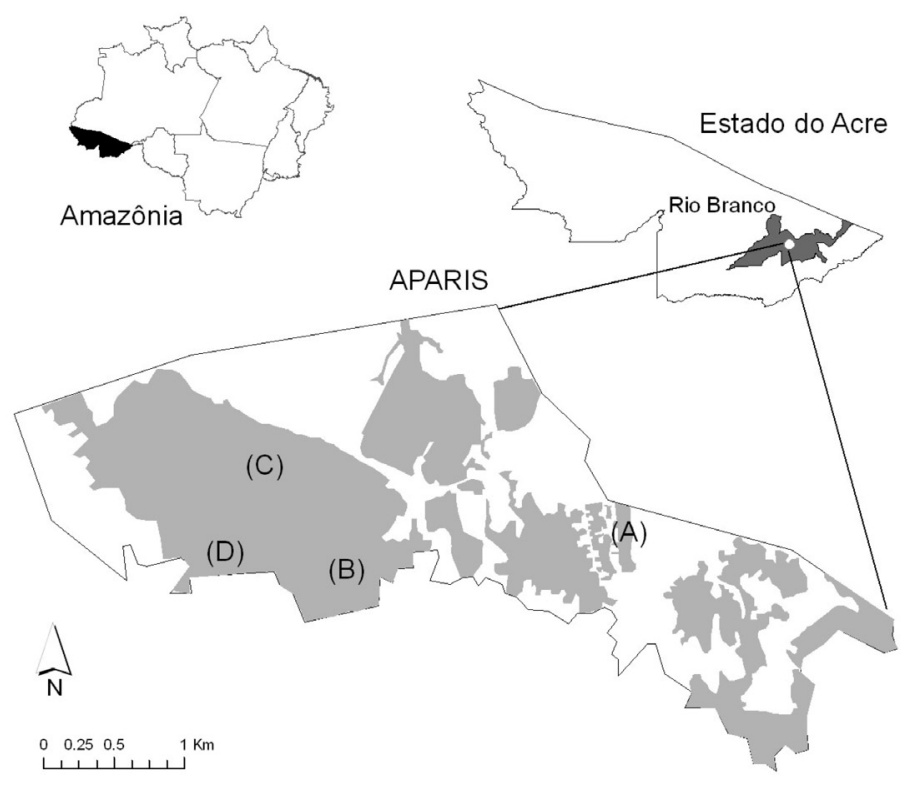

Figura 1 - Limites da Área de Proteção Ambiental Raimundo Irineu Serra- APARIS, localizada no município de Rio Branco - AC. Em cinza as áreas que apresentam cobertura vegetal e em branco as áreas sem cobertura vegetal. Localização das parcelas, onde: (A)-Capoeira 7,5 anos, (B)-Capoeira 27,5 anos, (C)-Capoeira 37,5 anos e (D)- Floresta primária

da vegetação de acordo com as espécies dominantes e altura média do dossel.

Em cada área de floresta secundária, e na área de floresta primária, foram instaladas cinco parcelas de 20 x $20 \mathrm{~m}$, totalizando 20 unidades amostrais distribuídas sistematicamente. Nas áreas com extensão suficiente, as parcelas foram distribuídas de forma alternada ao longo de um transecto de $100 \mathrm{~m}$ de comprimento. No caso de floresta primária, e da capoeira com 7,5 anos, onde a extensão de floresta contínua era inferior a $100 \mathrm{~m}$, as parcelas foram instaladas de acordo com a disponibilidade de espaço.

\section{Coleta e análise de dados}

Em cada parcela todos os indivíduos de palmeiras - desde plântulas a adultos, foram levantados e identificados. Os indivíduos foram classificados em cinco classes, segundo o tamanho e características vegetativo-reprodutivas, a saber: Classe 1: indivíduos de até $50 \mathrm{~cm}$ de altura; Classe 2: indivíduos com mais de $50 \mathrm{~cm}$ até $1 \mathrm{~m}$ altura, sem estipe aparente; Classe 3: indivíduos acima de $1 \mathrm{~m}$ de altura, sem estipe aparente; Classe 4: indivíduos com estipe aparente, náo-reprodutivos; Classe 5: indivíduos reprodutivos com cachos novos e/ou restos de cachos velhos, na planta ou no solo embaixo dos indivíduos.

A estrutura populacional foi avaliada separadamente para espécies acaulescentes (sem a presença estipe) e caulescentes (com estipe aparente) de acordo com as classes de tamanho descritas. No caso das espécies acaulescentes a quarta classe de altura foi desconsiderada.
Para análise de similaridade florística foi utilizado o índice de Jaccard. Os parâmetros fitossociológicos estimados, de acordo com Mueller-Dombois \& Ellenberg (1974), foram Densidades Relativa (DR) e Freqüências Relativa (FR). Foi calculado ainda o índice de diversidade de Shannon ( $\left.\mathrm{H}^{\prime}\right)$ e o índice de eqüitabilidade de Pielou (J), conforme descritos por Kent \& Coker (1992). Para análise dos dados utilizamos o software Mata Nativa 2.0.

\section{RESULTADOS E DISCUSSÃO}

\section{Composição florística}

Foram identificados 1.034 indivíduos, pertencentes a 12 gêneros e 19 espécies de palmeiras amostradas em 0,8 ha nas áreas de capoeira e floresta primária. Enquanto Rocha e Silva (2005), estudando as palmeiras de um fragmento de floresta secundária no município de Bragança, nordeste do estado do Pará, verificaram a ocorrência de nove gêneros e 14 espécies de palmeiras, em um universo de 504 indivíduos amostrados em 1,8 ha de florestas com diferentes tamanhos e idades, valor inferior ao encontrado no Acre.

Essa diferença pode ser influenciada pela diferença no histórico de uso das áreas, além do fato do decréscimo geral da diversidade de espécies na Amazônia no sentido oeste-leste, um fenômeno bem conhecido para muitos organismos. $\mathrm{O}$ decréscimo na diversidade de espécies na Amazônia central e ocidental em relação a sítios do oeste também é acompanhado da diminuiçáo na abundância relativa das espécies (FVA/ IBAMA, 1998). 
Quanto à distribuição de espécies identificadas na APARIS, observamos que: três ocorreram somente na área de floresta primária, sendo elas Euterpe precatoria, Geonoma acaulis e Socratea exorrhiza, oito somente nas áreas de capoeira com diferentes idades: Aiphanes aculeata, Bactris concinna, Bactris sp. 1, Bactris sp. 3, Geonoma sp. 2, Maximiliana maripa, Phytelephas macrocarpa, Syagrus sancona, e oito espécies ocorreram tanto em floresta primária quanto na secundária: Attalea phalerata, Astrocaryum ulei, Bactris maraja, Bactris sp. 2, Desmoncus polyacanthos, Oenocarpus mapora, Bactris bifida, Geonoma sp. 1, demonstrando alto grau de adaptação a diferentes ambientes.

Dentre as espécies restritas à floresta primária, $E$. precatoria e $S$. exorrhiza apresentam baixa resistência ao fogo (Henderson, 2002) - uma prática agrícola comum para a substituição da floresta por áreas agrícolas e de pastagens na regiáo. Fato este que pode ter contribuído para a ausência destas espécies nas áreas de florestas secundárias estudadas.

A. aculeata e $S$. sancona, duas espécies relativamente raras, demonstraram pouca capacidade de colonizar as diferentes capoeiras estudadas. No caso desta última, a presença de um adulto de grande porte, em estágio reprodutivo, sugere que o mesmo é, provavelmente, um remanescente da floresta antes da derrubada, tendo sido poupado e/ou resistido ao fogo que seguiu.

Espécies raras podem responder negativamente à proximidade de bordas (Matlack, 1994) sendo naturalmente mais vulneráveis devido sua baixa densidade. Hubbell e Foster (1986) ${ }^{1}$ apud Scariot (1998) sugerem que espécies raras têm maior grau de especialização de habitat e nicho de regeneração. Ainda segundo os mesmos autores, existe uma associaçáo ampla na comunidade, onde espécies raras tendem a ser especialistas e espécies comuns tendem a ser generalistas - em relaçáo à fase de regeneração em clareiras.

As espécies $A$. phalerata, A. ulei e B. maraja foram encontradas em todas as áreas amostradas, demonstrando grande resiliência e capacidade adaptativa, tanto para diferentes regimes de iluminação, quanto às alteraçôes antropogênicas ocorridas ao longo do tempo. B. maraja e Bactris sp. são consideradas espécies secundárias, que crescem em áreas abertas como clareiras (Scariot, 2000). A condiçâo de ser comum pode indicar que as espécies são resilientes à fragmentação.

Apesar do maior grau de resiliência e capacidade adaptativa das espécies observadas nas áreas secundária, a continuidade de açôes antrópicas ameaça a sobrevivência de muitas das espécies acima citadas. A ocorrência de fogo ou o corte raso nas áreas onde há uma pequena quantidade de indivíduos reprodutivos pode ocasionar a eliminação dos mesmos, diminuindo a produção de frutos e por conseqüência esgotando o banco de sementes.

Estes eventos não são incomuns na área da APARIS visto que a mesma encontra-se localizada em uma zona de transição urbano-rural onde a realizaçáo de desmatamentos ilegais por parte de moradores locais visando o erguimento de novas moradias e a ocorrência de queimadas nos fragmentos florestais adjacentes às áreas ocupadas por fazendas e lotes de pequenos agricultores vizinhos à APARIS, são observados de forma recorrente em razão da fiscalização deficiente da área.

Muitas vezes o fogo perde o controle e invade as áreas com floresta, causando mortalidade elevada, alteraçóes nos cíclos de nutrientes, e mundanças na composição florística, atrasando a acumulaçóes de biomassa em florestas secundárias e deixando-as ainda mais susceptível a queimadas futuras (Cochrane 2003, Zarin et al. 2005).

\section{Diversidade de espécies}

A diversidade florística determinada pelo Índice de Shannon $\left(H^{\prime}\right)$ variou de 0,35 a 1,65 , onde a maior diversidade de palmeiras foi encontrada nas parcelas instaladas na área de floresta primária, seguido pela capoeira com 27,5 anos (Tabela 1). No que se refere à eqüitabilidade, o maior valor também foi verificado na área de floresta $(\mathrm{J}=0,69)$, seguida pela capoeira com idade de 27,5 anos $(\mathrm{J}=0,63)$ (Tabela 1$)$.

Tabela 1- Índices de diversidade para as comunidades de palmeiras dos fragmentos de floresta secundária e primária da Área de Proteção Ambiental Raimundo Irineu Serra, Município de Rio Branco, AC, Brasil. N = número total de indivíduos; $S=$ número total de espécies na comunidade (riqueza); $\ln (\mathrm{S})$ = Logaritmo natural do número de espécies; $\mathrm{H}^{\prime}$ = Índice de Diversidade de Shannon-Wiener; $\mathrm{J}$ = índice de Eqüitabilidade de Pielou.

\begin{tabular}{lccccc}
\hline Área & $\mathrm{N}$ & $\mathrm{S}$ & $\ln (\mathrm{S})$ & $\mathrm{H}^{\prime}$ & $\mathrm{J}$ \\
\hline Capoeira 7,5 anos & 419 & 8 & 2,08 & 0,35 & 0,17 \\
Capoeira 27,5 anos & 128 & 10 & 2,3 & 1,44 & 0,63 \\
Capoeira 37,5 anos & 386 & 6 & 1,79 & 0,46 & 0,26 \\
Floresta Primária & 101 & 11 & 2,4 & 1,65 & 0,69 \\
\hline
\end{tabular}

A similaridade entre as áreas estudadas foi maior entre floresta primária e capoeira de 7,5 anos $(0,46)$, ou seja, os fragmentos com maior diferença de idade, e mais distantes espacialmente (Figura 1). Isto pode ser explicado pela presença de um banco de sementes com número maior de espécies conservadas e do número maior de espécies que apresentam rebrotamento através de rizoma. Os outros resultados

1 HUBBELL, S.P.; FOSTER, R.B. Commonness and rarity in a neotropical forest: implications for tropical tree conservation. In: SOULE, M.E., ed. Conservation biology: the science of scarcity and diversity. Suderland: Sinauer, 1986. p. 205-231. 
expressivos de similaridade foram verificados entre a área de floresta primária e a capoeira com 27,5 anos $(0,31)$ e 37,5 anos $(0,30)$ (Tabela 2). As condições ambientais similares como topografia e a drenagem, combinada com a proximidade do principal curso de água que atravessa a APARIS, podem explicar parcialmente a similaridade entre as áreas.

A área de capoeira com 37,5 anos apresentou, de uma maneira geral, baixa similaridade com as demais áreas e menor valor de riqueza de espécies. Essa condição pode ser parcialmente atribuída à ocorrência freqüente de perturbaçôes antropogênicas, como corte eventual da vegetação ou ocorrência recorrente de fogo, haja vista que o fragmento estudado é vizinho a uma pastagem cultivada. Esses eventos provavelmente impediram a sobrevivência de algumas espécies mais sensíveis às alteraçôes em seu habitat, especialmente aquelas do sub-bosque.

Tabela 2 - Índice de Similaridade de Jaccard entre os fragmentos de floresta secundária e primária da Área de Proteção Ambiental Raimundo Irineu Serra, Município de Rio Branco, AC, Brasil.

\begin{tabular}{lcccc}
\hline & $\begin{array}{c}\text { Capoeira } \\
7,5 \text { anos }\end{array}$ & $\begin{array}{c}\text { Capoeira } \\
27,5 \text { anos }\end{array}$ & $\begin{array}{c}\text { Capoeira } \\
37,5 \text { anos }\end{array}$ & $\begin{array}{c}\text { Floresta } \\
\text { Primária }\end{array}$ \\
\hline Capoeira 7,5 anos & 1,0 & & & \\
Capoeira 27,5 anos & 0,2 & 1,0 & & \\
Capoeira 37,5 anos & 0,17 & 0,14 & 1,0 & \\
Floresta Primária & 0,46 & 0,31 & 0,3 & 1,0 \\
\hline
\end{tabular}

A similaridade entre as áreas estudadas foi sempre inferior à metade do valor máximo de similaridade (Tabela 2) admitido pelo índice de Jaccard (Brower \& Zarr, 1984). As diferenças na composição dessas comunidades podem estar ligadas não somente as etapas sucessionais, mas a também a outros fatores como a intensidade e frequência do corte e queima da vegetação, e características ambientais relacionadas com o tipo de solo, gradiente topográfico e intensidade da luminosidade.

A baixa riqueza combinada com alta densidade de palmeiras na capoeira com 37,5 anos (Tabelas 1 e 3) sugeriu que a mesma foi submetida, no passado, a um ou vários eventos recorrentes de perturbação (fogo, derrubadas, abertura de clareiras para extraçáo de madeira, etc.), pois como observado e relatado pelos moradores locais, essa é uma prática comum na área. Isto pode ser corroborado pela semelhança com a capoeira mais nova ( 7,5 anos) que também demonstrou alta densidade de um número reduzido de espécies.

Além da fragmentação, Kahn \& de Granville (1992) apresentaram mais dois possíveis fatores para justificar a densidade de determinadas espécies de palmeiras em áreas de floresta tropical perturbadas. O primeiro relaciona-se ao fato das espécies serem úteis e por isso são poupadas durante a derrubada da floresta. Um segundo fator corresponde à maior resistência ao fogo; assim, durante as queimadas os indivíduos não são profundamente atingidos.

\section{Estrutura fitossociológica}

A espécie $A$. phalerata foi a mais densa em todas as áreas (Tabela 3), e foi uma espécie que observamos freqüentemente dominante em capoeiras da regiáo do estado do Acre em geral. A alta densidade de $A$. phalerata, principalmente nas áreas de capoeira mais jovens, se deve a suas características morfológicas que garantem a sua sobrevivência ao fogo e outras pertubaçóes. A sua forma de germinaçấo, do tipo remota-tubular, seguido por crescimento inicial que aterra a meristema apical ainda mais por baixo da superfície é outra estratégia usada pela espécie, em

Tabela 3- Parâmetros fitossociológicos das espécies de palmeiras encontradas nos fragmentos de floresta secundária e primária da Área de Proteção Ambiental Raimundo Irineu Serra, Município de Rio Branco, AC, Brasil. DR = Densidade relativa (\%);FR = freqüência relativa (\%).

\begin{tabular}{lcccccccc}
\hline & \multicolumn{2}{c}{7,5 anos } & & 27,5 anos & & 37,5 anos & \multicolumn{2}{c}{ Floresta Primária } \\
\cline { 2 - 9 } Espécie & FR & DR & FR & DR & FR & DR & FR & DR \\
\hline Attalea phalerata & 27,77 & 93,56 & 20,83 & 46,88 & 20,83 & 90,47 & 18,53 & 42,58 \\
Bactris maraja & 22,22 & 1,43 & 12,5 & 3,13 & - & - & 14,81 & 4,95 \\
Astrocaryum ulei & 5,56 & 0,24 & 20,83 & 28,13 & 16,67 & 1,59 & 18,53 & 27,72 \\
Phytelephas macrocarpa & 11,11 & 0,95 & 8,33 & 13,28 & 20,83 & 1,85 & - & - \\
Socratea exorrhiza & 11,11 & 2,15 & - & - & - & - & 14,81 & 5,94 \\
Euterpe precatoria & - & - & - & - & - & - & 11,12 & 7,92 \\
Oenocarpus mapora & 5,56 & 0,24 & 4,17 & 0,78 & - & - & 3,7 & 3,96 \\
Geonoma sp. 1 & - & - & - & - & - & - & 3,7 & 0,99 \\
\hline
\end{tabular}




\begin{tabular}{|c|c|c|c|c|c|c|c|c|}
\hline \multirow{2}{*}{ Espécie } & \multicolumn{2}{|c|}{7,5 anos } & \multicolumn{2}{|c|}{27,5 anos } & \multicolumn{2}{|c|}{37,5 anos } & \multicolumn{2}{|c|}{ Floresta Primária } \\
\hline & $\mathrm{FR}$ & $\mathrm{DR}$ & $\mathrm{FR}$ & $\mathrm{DR}$ & $\mathrm{FR}$ & $\mathrm{DR}$ & $\mathrm{FR}$ & DR \\
\hline Geonoma sp. 2 & - & - & - & - & - & - & - & - \\
\hline Maximiliana maripa & - & - & 4,17 & 0,78 & 12,5 & 1,59 & - & - \\
\hline Bactris concinna & 11,11 & 1,19 & - & - & - & - & - & - \\
\hline Desmoncus polyacanthos & - & - & - & - & 8,34 & 1,06 & 3,7 & 0,99 \\
\hline Geonoma acaulis & - & - & - & - & - & - & 3,7 & 0,99 \\
\hline Bactris bifida & 5,56 & 0,24 & - & - & - & - & 3,7 & 2,97 \\
\hline Aiphanes aculeata & - & - & 4,17 & 2,33 & - & - & - & - \\
\hline Syagrus sancona & - & - & 4,17 & 0,78 & - & - & - & - \\
\hline Bactris sp. 1 & - & - & 4,17 & 0,78 & - & - & - & - \\
\hline Bactris sp. 2 & - & - & 4,17 & 0,78 & - & - & 3,7 & 0,99 \\
\hline Bactris sp. 3 & - & - & 16,66 & 3,13 & 20,83 & 3,44 & - & - \\
\hline
\end{tabular}

seus estágios iniciais de desenvolvimento, tanto para sobreviver ao fogo quanto a danos mecânicos causados em sua parte aérea (Tomlinson, 1990; Anderson et al., 1991; Souza et al. 2000). Também, seu estipe geralmente é recoberto por bainhas foliares persistentes que funcionam como isolante das altas temperaturas em caso de fogo, e sua gema apical também está bem protegida por bainhas foliares de folhas vivas (McPherson \& Williams, 1998).

Dessa forma, quando uma floresta primária é derrubada e queimada, as plântulas dessa espécie, que possuem uma formidável capacidade de proteção e rebrotamento, encontram o ambiente ideal para se desenvolver. Um estudo realizado no município de Rio Branco mostrou que um ano depois do cortee-queima de uma área florestal para fins de cultivo agrícola, mais da metade das plântulas de $A$. phalerata (comprimento da folha $<2 \mathrm{~m}$ ), presentes na área antes da queima e da derrubada sobreviveu (Tucker Lima, dados ainda não-publicados).

Mesmo assim, enquanto a pertubação da floresta favorece espécies como $A$. phalerata, pode ser prejudical para outras espécies de palmeiras (Svenning 1998), e muitas dessas sequer regeneram em áreas abertas (Pedersen 1994, Moraes et al. 1995).

\section{Estrutura populacional}

Quando analisada a estrutura populacional das espécies caulescentes da capoeira com 7,5 anos de idade, a $1^{\text {a }}$ classe (plântulas) se destacou com mais da metade dos indivíduos amostrados (Figura 2).

Nas capoeiras de 27,5 e 37,5 anos, das espécies acaulescentes e caulescentes o maior percentual de indivíduos amostrados foi da $3^{\text {a }}$ classe de tamanho (Figuras 2 e 3). $\mathrm{Na}$ área de Floresta Primária, mais que a metade das palmeiras acaulescentes apresentou indivíduos na $5^{\mathrm{a}}$ classe, não ocorrendo nenhum indivíduo na $1^{\text {a }}$ e $3^{\mathrm{a}}$ classe de tamanho (Figura 3). A maior parte dos indivíduos das espécies caulescentes pertenceu a $2^{\mathrm{a}}$ classe de tamanho, com 45,74\% dos indivíduos amostrados, mas náo foram observados indivíduos da $5^{\text {a }}$ classe (Figura 2).

Entre os fragmentos avaliados, a capoeira mais jovem $(7,5$ anos) apresentou uma distribuição de classes de tamanho com um número maior de plântulas, seguindo o padrão-J invertido interpretado como um indicador de estabilidade ou incremento populacional (Martini, 1996). Vale ressaltar, entretanto, que esta capoeira apresentou menor diversidade de palmeiras. As demais áreas apresentaram um número reduzido de indivíduos adultos em fase reprodutiva, e baixo número de plântulas recrutadas.

O número baixo de plântulas de palmeiras pode ter sido influenciado pela falta de indivíduos reprodutivos e pelo isolamento dos fragmentos das áreas mais velhas, que podem servir como fontes de semente. Outros eventos atípicos, como queimadas acidentais, podem estar eliminando as plântulas do local. De acordo com Scariot (2000), o número total de palmeiras numa área de floresta é grandemente influenciado pela quantidade de plântulas, que é o estágio de vida mais prejudicado pela fragmentação. Este estágio de vida das palmeiras é também aquele com a maior taxa natural de mortalidade (Vargas, 2004).

A área de floresta primária amostrada consiste em uma área pequena (área de proteção permanente) cercada por 

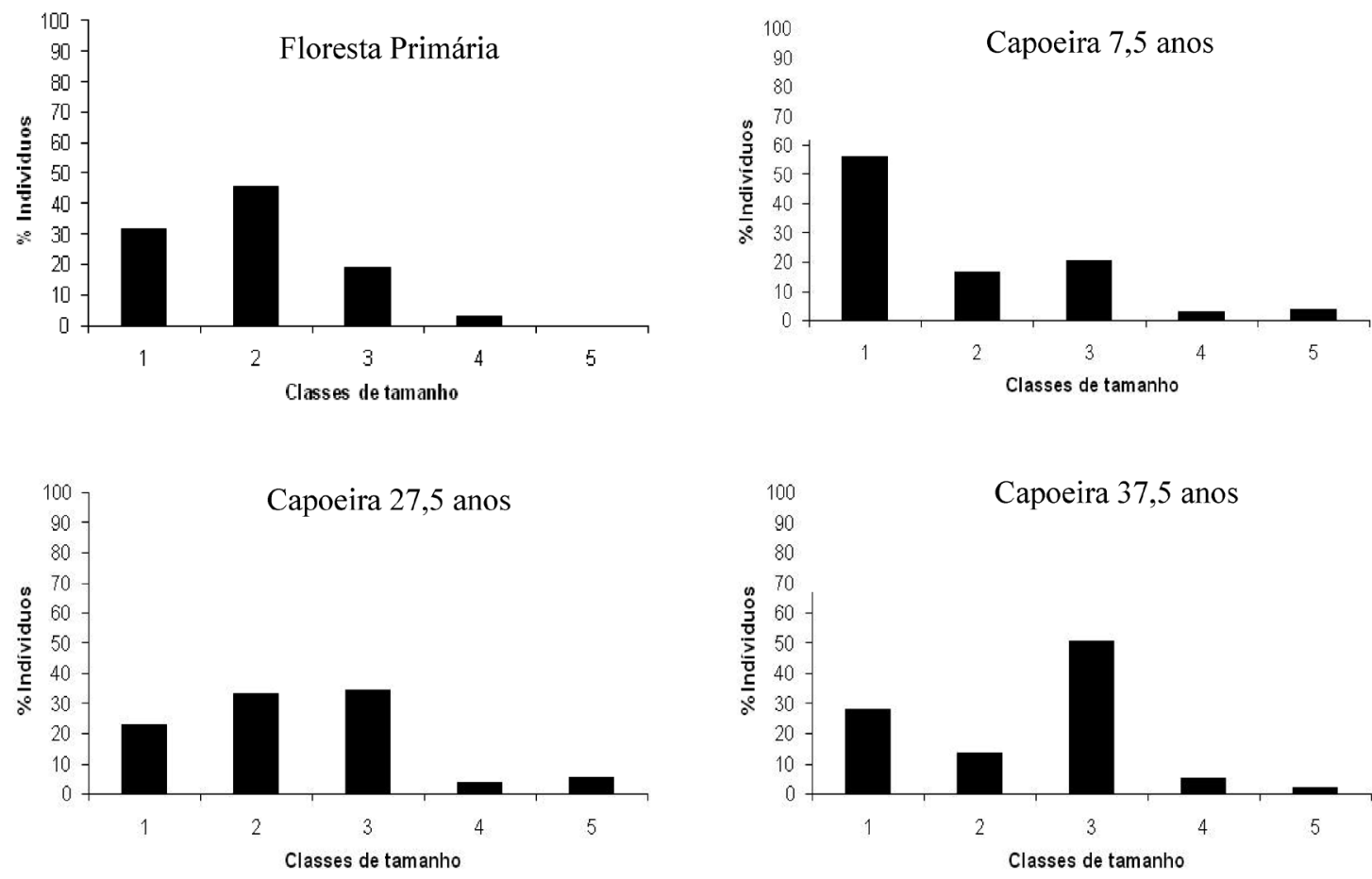

Figura 2- Estrutura populacional das comunidades de palmeiras caulescentes amostradas nas parcelas da Área de Proteção Ambiental Raimundo Irineu Serra, Município de Rio Branco, AC. Classe $1=$ indivíduos $\leq 50 \mathrm{~cm}$ de altura; Classe 2 = indivíduos $50 \mathrm{~cm}-1 \mathrm{~m}$ de altura; Classe $3=$ indivíduos $>1 \mathrm{~m}$ de altura sem estipe aparente, não reprodutivo; Classe $4=$ indivíduos $>1 \mathrm{~m}$ de altura com estipe aparente, não reprodutivo; Classe $5=$ Indivíduos reprodutivos.
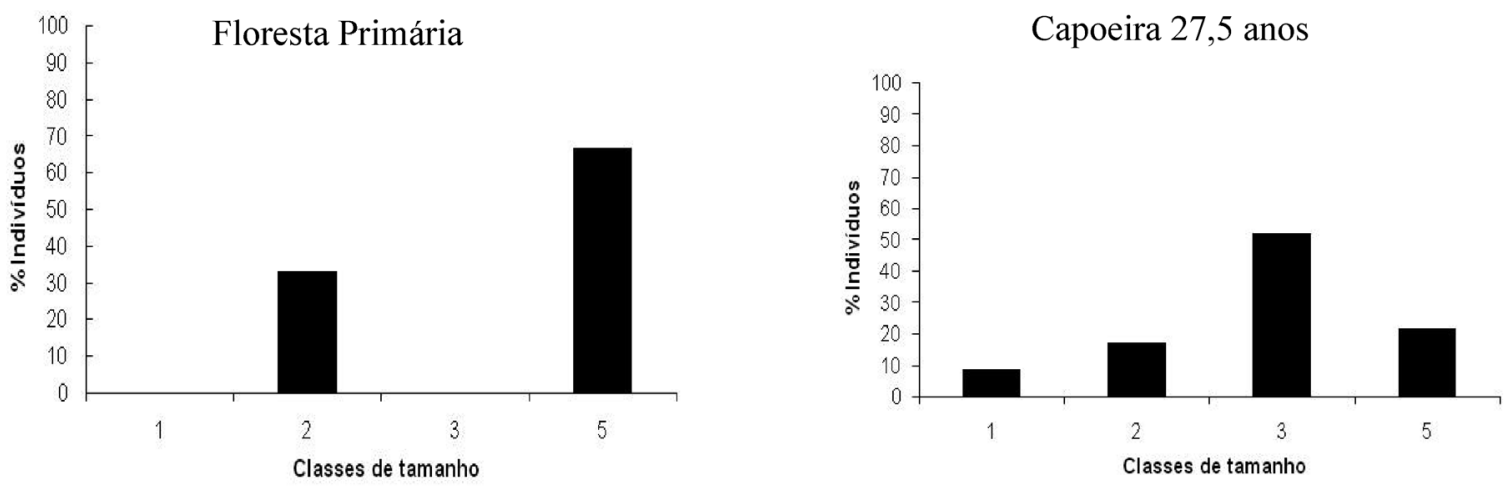

Capoeira 37,5 anos

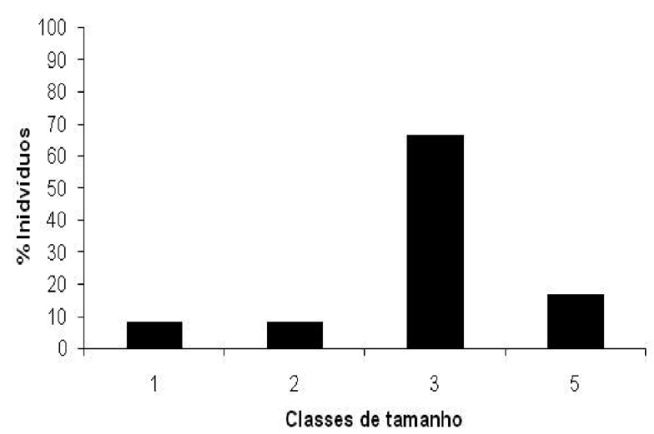

Figura 3 - Estrutura populacional das comunidades de palmeiras acaulescentes amostradas nas parcelas da Área de Proteção Ambiental Raimundo Irineu Serra, Município de Rio Branco, AC. Classe $1=$ indivíduos $\leq 50 \mathrm{~cm}$ de altura; Classe 2 = indivíduos $50 \mathrm{~cm}-1 \mathrm{~m}$ de altura; Classe $3=$ indivíduos $>1 \mathrm{~m}$ de altura sem estipe aparente, não reprodutivo; Classe $5=$ Indivíduos reprodutivos. 
floresta secundária, representando menos de $5 \%$ da área florestal existente na unidade de conservação. Este isolamento pode estar agindo negativamente na vitalidade original da comunidade, tanto pela eliminaçấo de adultos reprodutivos como pela mudança das condiçóes ambientais, que resultaram em uma alteração significativa da diversidade de palmeiras. Isto pode ser confirmado pela ausência de indivíduos em determinadas classes de tamanho na estrutura da população de algumas espécies típicas de floresta primária, como $E$. precatoria, O. mapora e S. exorrhiza.

O tamanho das parcelas e o tipo de amostragem utilizada podem ter excluído alguns indivíduos em razão dos seus padróes de distribuiçáo espacial visto que estudos sobre comunidades vegetais podem não detectar as conseqüências severas da fragmentação em populaçôes específicas de espécies que não são uniformemente distribuídas (Scariot, 1998).

O re-estabelecimento de espécies de palmeiras originalmente existentes nas áreas, nem sempre segue padrôes pré-definidos, porque a formação e o desenvolvimento da comunidade dependem tanto de fatores físicos e bióticos como tipo de solo e distância de fontes de propágulo, quanto do histórico e da intensidade de uso da área (Mesquita et al., 2001).

A fragmentação também afeta a diversidade e a abundância da fauna local, incluindo aqueles animais que agem como predadores e dispersores das sementes de palmeiras, com efeitos diretos no recrutamento de novos indivíduos e na dispersão das sementes (Pimentel e Tabarelli, 2004).

Espécies como $A$. phalerata, que possuem sementes mecanicamente mais resistentes, são beneficiadas com o desaparecimento de seus predadores especializados (roedores). Isto geralmente leva a uma situaçáo de alta taxa de recrutamento de plântulas e dominância da espécie em uma dada área, como temos observado neste estudo.

\section{CONCLUSÃO}

Os resultados confirmados neste estudo comprovam uma grande variação na composição e estrutura das populações de palmeiras em florestas secundárias e primárias. A fragmentação decorrente da derrubada e a ocorrência de perturbaçôes derivadas de queimadas e abertura de trilhas no interior dos fragmentos florestais alteraram a composição e diminuíram a riqueza e a diversidade de palmeiras na área da APARIS, e ao mesmo tempo, estão favorecendo a dominância de certas espécies como $A$. phalerata.

As espécies de palmeiras respondem de maneira diferente ao avanço do tempo de regeneração na capoeira. Enquanto o fogo e o corte raso excluem palmeiras de todos os tamanhos, a eliminação específica de indivíduos em estágio reprodutivo e a falta de áreas de floresta primária estão limitando a disponibilidade de sementes e, conseqüentemente, o recrutamento de novas plântulas.

A escassez de plântulas e adultos reprodutivos para a maioria das espécies avaliadas indica uma possível ameaça para sobrevivência de grande parte das espécies de palmeiras nessa área ao longo dos anos. Embora, algumas dessas espécies sejam mais resistentes a antropização e capazes de se adaptar facilmente em novos ambientes possuem maiores vantagens para sobrevivência nessas áreas alteradas, criando uma tendência para uma floresta dominada por uma ou poucas espécies.

\section{BIBLIOGRAFIA CITADA}

Acre. Governo do Estado do Acre. 2000. Programa Estadual de Zoneamento Ecológico-Econômico do Estado do Acre. Ecological-Economical Zoning: natural resources and environment - final document. V.1. SECTMA, Rio Branco, Acre (in Portuguese).

Anderson, A.B.; May, P.H.; Balick, M.J. 1991. The Subsidy from Nature: Palm Forests, Peasantry, and Development on an Amazon Frontier. Columbia University Press, New York, USA. 233 pp.

Bierregaard, R.O.T.; Lovejoy, T.E.; Kapos, V; Santos, A.; Hutchings, R. 1992. The biological dynamics of tropical rain forest fragments. Bioscience, 42: 859-866.

Brower, J.E.; Zarr, J.H. 1984. Field and laboratory methods for general ecology. W. C. Brown Publishers, Dubuque, Iowa, USA. 226 pp.

Brown, J.H. 1984. On the relationship between abundance and distribution of species. American Naturalist, 124: 253-279.

Cochrane, M.A. 2003. Fire science for rainforests. Nature, 421: 913-919.

Ferreira, E.J.L. 2005. Manual to the palms of Acre, Brazil. (http:// www.nybg.org/bsci/acre/www1/manual_palmeiras.html). Acesso: 10/01/2007 (in Portuguese).

FVA/IBAMA, 1998. Management Plan for Jau National Park. Fundação Vitória Amazônica, Manaus, Amazonas. 258 pp (in Portuguese).

Henderson, A. 2002. Evolution and ecology of palms. New York Botanical Garden Press. Bronx, NY, USA. 198 pp.

Kahn, F.; de Granville, J.J. 1992. Palms in forest ecosystems of Amazonia. Springer-Verlag, Berlin. 226 pp.

Kent, M.; Coker, P. 1992. Vegetation description and analysis: A practical approach. John Wiley \& Sons, Chichester, UK. 384 pp.

Lovejoy, T.E.; Bierregaard, R.O.; Rylands, A.B.; Malcolm, J.R.; Quintela, C.E.; Harper, L.H.; Brown, K.S.; Powell, A.H.; Powell, G.V.N.; Schubart, H.O. R.; Hays, M.B. 1986. Edge and other effects of isolation on Amazon forest fragments, p. 257-285. In: Soulé, M.E. (Ed). Conservation Biology: The Science of Scarcity and Diversity. Sinauer, Sunderland, Massachusetts, USA.

Martini, A.M.Z. 1996. Structure and population dynamics of three tropical tree species. Dissertação de Mestrado. Universidade Estadual de Campinas, Campinas, Amazonas (in Portuguese). 
Matlack, G.R. 1994. Vegetation dynamics of the forest edge: trends in space and successional time. Journal of Ecology, 82: 113-123.

McPherson, K.; Williams, K. 1998. The role of carbohydrate reserves in the growth, resilience, and persistence of cabbage palm seedlings (Sabal palmetto). Oecologia, 117: 460-468.

Mesquita, R.C.G.; Ickes, K.; Ganade, G.; Williamson, G.B.. 2001. Alternative successional pathways in the Amazon basin. Journal of Ecology, 89: 528-537.

Mueller-Dumbois, D.; Ellenberg, H. 1974. Aims and methods vegetation ecology. John Wiley \& Sons, New York, NY, USA. $547 \mathrm{pp}$.

Murcia, C. 1995. Edge effects in fragmented forests: implications for conservation. Trends in Ecology and Evolution, 10: 58-62.

Pedersen, H.B. 1994. Management, extractivism and commercial use of wild palms in Ecuador, p. 12-22. In: Rios, M.; Petersen, H.B. (Eds). Las Plantas y el Hombre. ABYA-YALA, Quito, Ecuador.

Pimentel, D.S.; Tabarelli, M. 2004. Seed Dispersal of the Palm Attalea oleifera in a Remnant of the Brazilian Atlantic Forest. Biotropica, 36: 74-84.

Pinto, S.R. 2007. Restructuring of the palm community (Arecaceae) in a secondary forest of the Central Amazon. 9 pp. (http:// tamandua.inpa.gov.br/ $\sim$ pdbff/cursos/efa/livro/2007/pdf/km41/ final_severino.pdf) Acesso: 30/03/2009 (in Portuguese).

PMRB, 2005. Request for the creation of the Raimundo Irineu Serra Environmental Protection Area (APA), Rio Branco, Acre. Secretaria Municipal de Meio Ambiente da Prefeitura Municipal de Rio Branco. 54 pp (in Portuguese).

Rocha, A.E.S.; da Silva, M.F.F. Phytosociological, floristic, and ethnobotanical aspects of palms (Arecaceae) in secondary forest in Bragança, PA. Acta Botanica Brasilica, 19: 657-667 (in Portuguese).

Scariot, A. 2000. Seedling mortality by litterfall in Amazonian forest fragments. Biotropica, 32: 662-669.

Scariot, A. 1998. Consequences of forest fragmentation on palm communities in Central Amazonia. SérieTécnica IPEF, 12: 7186 (in Portuguese).
Shafer, M.L. 1981. Minimum population sizes for species conservation. Bioscience, 31: 131-134.

Souza, A.F.; Martins, F.R.; Matos, D.M.S. 2000. Detecting ontogenetic stages of the palm Attalea humilis in fragments of the Brazilian Atlantic forest. Canadian Journal of Botany, 78: 1227-1237.

Svenning, J.C. 1998. The effect of land-use on the local distribution of palm species in an Andean rain forest fragment in northwestern Ecuador. Biodiversity and Conservation, 7: 1529-1537.

Terborgh, J.; Lopes, L.; Tello, J.; Yu, D.; Bruni, A.R. 1997. Transitory states in relaxing ecosystems of land bridge islands, p. 256274. In: Laurance, W.F.; Bierregaard, R.O. (Eds). Tropical Forest Remnants: Ecology, Management, and Conservation of Fragmented Landscape. University of Chicago Press, Chicago, USA.

Tilman, D.; May, R.M.; Lehman, C.L.; Nowak, M.A. 1994. Habitat destruction and the extinction debt. Nature, 371: 65- 66.

Tomlinson, P.B. 1990. The structural biology of palms. Oxford University Press, New York, NY, USA. 492pp.

Vargas, M.P.B. 2004. Demography of Attalea butyracea in the Bolivian Amazon. Master's Thesis, Utrecht University.

Zarin, D.J.; Davidson, E.A.; Brondizio, E.; Vieira, I.C.G.; Sá, T.: Feldpausch, T.; Schuur, E.A.G.; Mesquita, R.; Moran, E.; Delamonica, P.; Ducey, M.J.; Hurtt, G.C.; Salimon, C.; Denich, M. 2005. Legacy of fire slows carbon accumulation in Amazonian forest regrowth. Frontiers in Ecology and the Environment, 3: 365-369.

Zudeima, P.A.; Sayer, J.A.; Dijkman, W. 1996. Forest fragmentation and biodiversity: the case for intermediate-sized conservation areas. Environmental Conservation, 23: 290-297.

Recebido em 15/02/2009

Aceito em 25/11/2009 
